Revista de Psicología Vol. 36 (1), 2018 (ISSN 0254-9247)

\title{
Interacción sociocognitiva entre pares en situaciones simétricas $y$ asimétricas de competencia epistémica ${ }^{1}$
}

\author{
Mariano Castellaro², Néstor Roselli ${ }^{3}$ \\ Consejo Nacional de Investigaciones Científicas y Técnicas (CONICET) $)^{1,2}$, \\ Universidad Nacional de Rosario ${ }^{1}$, Pontificia Universidad Católica Argentina ${ }^{2}$
}

\begin{abstract}
El objetivo del estudio fue analizar la interacción sociocognitiva entre pares y su relación con el avance cognitivo individual, en situaciones de simetría y asimetría de competencia epistémica. Participaron 72 estudiantes de quinto y sexto grado $(M=11$ años, 3 meses; $D E=7$ meses), quienes resolvieron ítems lógicos (pre/post prueba individual, condición diádica intermedia). Los resultados indicaron: a) la distribución del aporte argumental en la díada es el principal diferenciador procesual entre las condiciones simétricas analizadas; b) el volumen y distribución de los argumentos de la díada se asocian significativamente con el avance cognitivo individual, independientemente de la condición de simetría/asimetría de competencia epistémica. Los resultados se discuten a la luz de los fundamentos socioconstructivistas del estudio.

Palabras clave: Interacción sociocognitiva, colaboración entre pares, simetría epistémica, asimetría epistémica, aprendizaje colaborativo.
\end{abstract}

1 Los autores expresan el más sincero agradecimiento para las autoridades, padres y alumnos de las escuelas donde se realizó esta investigación. Sin su colaboración esta investigación no hubiera sido posible. También se destaca la importante participación de los estudiantes Lucía Arjol, Juan Manuel Curcio y Nicolás Morguen en el relevamiento y codificación de los datos.

2 Doctor en Psicología. Investigador del Consejo Nacional de Investigaciones Científicas y Técnicas (CONICET), Argentina. Lugar de trabajo: Instituto Rosario de Investigaciones en Ciencias de la Educación (IRICE-UNR). Docente de la cátedra Metodologías y Gestión de la Investigación en Psicología, Carrera de Psicología, Facultad de Psicología de la Universidad Nacional de Rosario (UNR), Rosario, Argentina. Dirección postal: 27 de febrero 210 bis (Ocampo y Esmeralda), Rosario (Santa Fe), Argentina (C.P. 2000). Contacto: castellaro@ irice-conicet.gov.ar

3 Doctor en Psicología. Investigador del Consejo Nacional de Investigaciones Científicas y Técnicas (CONICET), Argentina. Director del Centro de Investigaciones en Psicología de la Facultad de Psicología y Psicopedagogía de la Pontificia Universidad Católica Argentina (UCA), Buenos Aires, Argentina. Dirección postal: A. M. de Justo 1300, Buenos Aires, Argentina (C.P. 1107). Contacto: nestorroselli@uca.edu.ar 


\section{Peer sociocognitive interaction according to symmetry and asymmetry of epistemic competence}

The study aimed to analyze the peer sociocognitive interaction and its relationship with individual cognitive progress, in both symmetrical and asymmetrical situations of epistemic competence. Participants were 72 students of fifth and sixth grade (age, $M=11$ years, 3 months, $S D=7$ months), who solved logical items (pre / post test individual, intermediate dyadic condition). The results indicated: a) the distribution of arguments in the dyad is the main variable of the interactive process related to differences between symmetrical conditions and b) the volume and distribution of the arguments in dyads are significantly associated with individual cognitive advancement, independently of symmetrical/asymmetrical condition. The results are discussed from a socioconstructivist perspective.

Keywords: Sociocognitive interaction, peer collaboration, epistemic symmetry, epistemic asymmetry, collaborative learning.

\section{Interaçáo sociocognitiva entre pares em situaçóes simétricas e assimétricas de compe- tência epistêmica}

O objetivo do estudo foi analisar a interação sociocognitiva entre pares e sua relação com o progresso cognitivo individual, em situaçóes de simetria e assimetria de competência epistêmica. Participaram 72 alunos do quinto e sexto ano (idade, $M=11$ anos, 3 meses, $D E=7$ meses), que resolveram itens lógicos (pré / pós-teste individual, condição diadêmica intermediária). Os resultados indicaram: a) a distribuição da argumentação na díade é o principal diferencial processual entre as condiçóes simétricas analisadas; b) o volume e a distribuição dos argumentos da díade estão significativamente associados ao avanço cognitivo individual, independentemente da condição de simetria / assimetria de competência epistêmica. Os resultados são discutidos à luz dos fundamentos socioconstrutivistas do estudo.

Palavras-chave: Interação sociocognitiva, colaboração entre pares, simetria epistêmica, asimetria epistêmica, aprendizagem colaborativa.

\section{Interaction socio-cognitive entre paires dans des situations symétriques et asymétriques de compétence épistémique}

L'objectif de l'étude était d'analyser l'interaction sociocognitive entre pairs et leur relation avec le progrès cognitif individuel, dans des situations de symétrie et d'asymétrie de la compétence épistémique. 72 élèves de cinquième et sixième année ont participé ( $M=11$ ans, 3 mois, $D E=7$ mois), qui ont résolu des items logiques (test pré / post individuel, condition dyadique intermédiaire). Les résultats ont indiqué: a) la distribution de la contribution dans l'argumentation dans le couple est le principal différentiateur procédural entre les conditions symétriques analysées; b) le volume et la distribution des arguments de le couple sont significativement associés à l'avance cognitive individuelle, indépendamment de la condition de symétrie / asymétrie de la compétence épistémique. Les résultats sont discutés sous l'angle des fondements socioconstructivistes de l'étude.

Mots-clés: interaction socio-cognitive, collaboration entre pairs, symétrie épistémique, asymétrie épistémique, apprentissage collaborative. 
El socioconstructivismo sostiene el rol constructivo de las interacciones entre pares en el desarrollo cognitivo y/o construcción de conocimientos, sobre todo en comparación con el trabajo individual o solipsista (Gilly, 1992; Psaltis, Duveen \& Perret Clermont, 2009). Más allá de esta premisa general, no es posible afirmar taxativamente una relación directa entre trabajo colectivo y desarrollo individual, debido a la presencia de diversos factores mediadores.

Uno de dichos factores, el cual ha recibido especial atención dentro de una psicología social del desarrollo cognitivo (Mugny \& Pérez, 1988), es el nivel de competencia específica de los compañeros de trabajo, puntualmente, el grado de similitud (o disimilitud) relativo entre estas. El interés por la cuestión se fundamenta teóricamente en que, considerando que la interacción social es estudiada en relación al desarrollo de una habilidad determinada y, por consiguiente, en el marco de una tarea puntual, es postulable que el grado de igualdad/desigualdad entre las competencias específicas de los compañeros determine una particular modalidad interactiva la que, a su vez, determinaría un particular grado de avance cognitivo individual.

Históricamente, este asunto fue discutido desde dos perspectivas: neopiagetiana y neovygotskiana. Aunque ambos enfoques han enfatizado la importancia de alguna forma de desigualdad entre los sujetos, es posible reconocer algunos desacuerdos más sutiles entre estas. En primer lugar, en relación a la forma de conceptualizar esa diferencia entre los sujetos (Asterhan, Schwarz \& Cohen-Eliyahu, 2014), las posiciones neopiagetianas privilegian el intercambio entre individuos con similares niveles de competencia inicial, aunque con diferentes perspectivas sobre un mismo problema, condición básica inicial para el conflicto sociocognitivo (diferencias cualitativas). Las posiciones neovygostkianas, por su parte, enfatizan el valor de la asimetría en lo que 
hace al grado de competencia, especialmente para los sujetos menos competentes que interactúan con un compañero más avanzado, lo cual se asocia con el concepto clásico de zona de desarrollo próximo (de aquí en adelante, $\mathrm{ZDP})$.

En segundo lugar, en relación a la interacción entre sujetos igualmente deficitarios, algunos trabajos clásicos más emparentados con un enfoque piagetiano sostienen la posibilidad de progreso cognitivo individual, por ejemplo, entre niños no conservadores (Light, Littleton, Messer \& Joiner, 1994; Doise \& Mugny, 1984; Perret Clermont, 1980; Roselli, Gimelli \& Hechen, 1995). Desde el marco de la teoría del conflicto sociocognitivo, este dato es de suma relevancia ya que confirmaría el hecho de que el desarrollo cognitivo es posible a partir de la interacción entre pares en el sentido más puro del término, y no por un factor de tutoría tal como el propuesto desde los modelos neovygotskianos. Sin embargo, otros estudios basados en la teoría sociocultural han arribado a conclusiones diferentes que no avalan la hipótesis del progreso cognitivo construido desde la igualdad deficitaria (los cuales se citarán más adelante).

Ambas líneas socioconstructivistas parten de un diseño metodológico más o menos similar, aunque — por supuesto — en cada caso adquiere características propias. Básicamente está constituido por tres fases principales. Primero, un pretest del grado de competencia individual en relación a una tarea o dominio puntual (en algunos casos también hay un pretest del grado de competencia colectivo, por ejemplo, los trabajos de Neil Mercer que se comentarán más arriba). Segundo, una fase interactiva de trabajo conjunto sobre un problema (por ejemplo, entre dos niños). Aquí se pueden integrar compańeros con perspectivas diferentes sobre un problema (sobre todo en el caso de las perspectivas piagetianas) o compañeros con diferentes grados de competencia (especialmente en el caso de las perspectivas neovygotskianas). En algunos trabajos, esta fase intermedia consiste en un programa de entrenamiento en aprendizaje colaborativo (por ejemplo, Learning Together desarrollado por Mercer y colaboradores). Por último, la fase de postest individual (o también colectivo, según la 
investigación) se utiliza para evaluar los efectos de la interacción sobre el grado de competencia individual inicial (pretest).

La línea mayormente inspirada en un enfoque crítico de la psicología genética piagetiana privilegió sus estudios en torno a la construcción de la noción de conservación, o sea, analizando interacciones entre dos niños no conservadores, entre dos niños conservadores, o entre un niño conservador y otro no conservador. Se analizaron tareas adaptadas de las propuestas originalmente por Piaget (por ejemplo, Piaget, 1923/2002), como ser trasvasamiento de líquido (Doise \& Mugny, 1984; Psaltis \& Duveen, 2006, 2007) o rotación de planos (Doise \& Mugny, 1984; Psaltis, 2011; Zapiti \& Psaltis, 2012). Otra variante dentro de esta línea son los trabajos sobre desarrollo moral, distinguiendo entre niños heterónomos y autónomos (por ejemplo, Leman \& Duveen, 1999, 2003).

Desde este enfoque, la interacción entre individuos con diferentes perspectivas sobre un mismo problema es una condición necesaria, más no suficiente, para que se origine un auténtico conflicto sociocognitivo (motor básico del desarrollo intelectual). No solo depende de que cada compañero cuente con los prerrequisitos cognitivos básicos (Gilly, 1992), sino fundamentalmente de la forma en que dicha disidencia entre los puntos de vista individuales es resuelta por los compañeros de trabajo. Así, se han distinguido dos formas básicas de resolución del conflicto, siendo únicamente la primera la conducente al avance cognitivo individual: a) epistémica: basada en la confrontación simétrica entre posiciones individuales y la búsqueda de una solución cognitiva común en base a argumentaciones y justificaciones lógicas; b) relacional: basada en la comparación entre las posiciones sociales de los participantes y una decisión final por imposición o confrontación competitiva (Buchs, Butera, Mugny \& Darnon, 2004; Peralta \& Roselli, 2016; Peralta, Roselli \& Borgobello, 2012; Quimzade \& Mugny, 2001).

De manera complementaria, Psaltis (2011), Psaltis \& Duveen (2006, 2007) y Zapiti \& Psaltis (2012), en una tarea de rotación de planos en niños de cinco y seis años, diferenciaron cuatro formas 
básicas de resolución del conflicto, siendo la primera de ellas la única que auténticamente conduce al avance cognitivo individual del sujeto inicialmente no conservador: Reestructuración explícita (el sujeto no conservador logra construir activamente una solución conservadora del problema, lo cual es manifestado verbalmente durante la interacción); No conservación (el niño no conservador logra persuadir al conservador); No resistencia (el sujeto no conservador pasivamente acepta el argumento del compañero conservador, sin atender al punto de vista propio); Resistencia (el sujeto no conservador persevera en su propio punto de vista y rechaza la propuesta del compañero conservador).

Por otra parte, desde una perspectiva más integrativa entre las posiciones piagetiana y vygotskiana, Jonathan Tudge (Tudge, 1989, 1992; Tudge \& Winterhoff, 1993; Tudge, Winterhoff \& Hogan, 1996) propuso otra actividad para estudiar la cuestión, en niños de entre cinco y nueve ańos: una tarea de equilibrio (Balance Beam Task). Se trata de una balanza con cuatro puntos simétricos en cada brazo (cuatro distancias regulares respecto al centro), sobre los cuales se pueden colocar cantidades diversas de aros de metal. La actividad requiere que los niños infieran si ambos brazos se mantendrán en equilibro o no (y en ese caso, en qué dirección), en función de los pesos que se distribuyan a cada lado. Sobre esta tarea, se diferenciaron seis reglas (niveles) de resolución del problema, ordenadas progresivamente de un menor a un mayor entendimiento de la noción lógica de balance. En este caso, el análisis de la simetría y asimetría cognitiva se basó en la conformación de díadas integradas por niños que habían utilizado, respectivamente, similares o diferentes reglas de solución en el pretest (con un máximo de dos niveles de desigualdad entre estas). En términos generales, estos trabajos concluyeron que los principales beneficiados de la colaboración son los nińos de baja competencia que interactuaron con un compañero de mayor habilidad. Sin embargo - lo cual es muy relevante- también se detectaron retrocesos en las performances individuales de algunos individuos que habían participado en díadas asimétricas, tratándose especialmente de aquellos de mayor grado de competencia inicial. 
Denessen, Veenman, Dobbelsteen \& Van Schilt (2008) retomaron esa misma tarea de equilibrio utilizada anteriormente, aunque únicamente en díadas asimétricas de niños de 11 y 12 años. Se destacaron dos modificaciones en el diseño: a) el reconocimiento de tres niveles de competencia (baja, media y alta) en lugar de los dos propuestos previamente; b) dicho nivel de competencia no fue determinado por una evaluación inicial individual relativa a la propia tarea de balance, sino a un formato de evaluación estándar en matemáticas desarrollado por un instituto nacional gubernamental. El análisis de los datos se enfocó principalmente en los efectos de la composición de la díada (bajo-medio y medio-alto) sobre el grado de participación y elaboración cognitiva durante el trabajo colaborativo. Si bien las conclusiones refieren a la performance de los niños durante la interacción (y no al avance individual consecuente), contrariamente a los antecedentes previos, los autores sostienen que los individuos de mayor habilidad, es decir, los sujetos de nivel medio o alto que trabajan con un niño de nivel bajo o medio (respectivamente), son los principales beneficiados de la colaboración, sobre todo en el primer caso (medio con bajo).

Por su parte, Fawcett \& Garton (2005) y Garton \& Pratt (2001) concluyeron en el mismo sentido de los trabajos de Tudge (los principales beneficiados son los niños de nivel bajo que trabajan con un compañero más competente), aunque en relación a otro tipo de tarea y con niños sujetos más pequeños (cuatro y siete años). Se trató de una tarea de clasificación (sorting task), donde los materiales suponían un conjunto de objetos con una serie de características diferenciales que permitían generar distintas agrupaciones entre sí (por ejemplo, bloques de tres colores, dos formas, dos tamańos y dos anchos). La consigna solicitaba generar la mayor cantidad posible de formas de clasificación de dichos objetos, ya sea en base a un único criterio (por ejemplo, por color) o combinando dos o más de estos (por ejemplo, según color y forma).

Schmitz y Winskel (2008) analizaron la interacción en díadas con asimetría de competencias en nińos de entre diez y doce años, en la 
resolución de problemas matemáticos, diferenciando tres niveles de competencia: bajo, medio y alto. Se conformaron díadas integradas por niños de nivel bajo y medio, por un lado, y díadas integradas por niños de nivel bajo y alto, por el otro, para analizar la influencia del grado de desigualdad cognitiva sobre la interacción. En las díadas con asimetría moderada (bajo - medio) se registró un mayor lenguaje exploratorio (Mercer, 2001), en comparación con las díadas con asimetría extrema (bajo-alto). Los autores concluyeron que esto se da porque una mayor cercanía cognitiva permite un sostenimiento mutuo en la resolución de la tarea. En este sentido, la asimetría extrema deriva en la dominación, aburrimiento o pérdida de paciencia por parte del sujeto de mayor habilidad y/o la disociación de la tarea por parte del niño menos competente.

También Asterhan et al. (2014) analizaron la cuestión de la simetría/asimetría epistémica, trabajando con estudiantes de 14 y 15 ańos de edad, en una tarea de razonamiento proporcional. Primero, los sujetos observaban en una lámina una serie de cuatro bloques tridimensionales (A, B, C y D), cada uno constituido por una cantidad diferente de ladrillos (todos del mismo tamańo y forma). Los ladrillos de los bloques A y C tenían el mismo color y peso; lo mismo sucedía entre los ladrillos de los bloques B y D. Sobre esa base, los individuos debían resolver una serie de ítems de razonamiento proporcional. En cada subprueba, se les daba información sobre una posible relación entre los bloques A y B (por ejemplo, que A era más pesado que B). A partir de ello, debían deducir la relación entre $\mathrm{C}$ y $\mathrm{D}$ y justificar verbalmente la solución propuesta. En el marco de esa prueba, los investigadores propusieron cuatro niveles crecientes de razonamiento proporcional: nivel 1 de carácter rudimentario (sujetos con esa respuesta fueron descartados para el estudio); niveles 2 y 3 erróneos, aunque basados en estrategias diferentes; nivel 4 acertado y basado en una lógica correcta.

El aporte metodológico de este estudio consistió en el uso de la combinación entre los tres niveles superiores para analizar las hipótesis neopiagetiana y neovygotskiana: en relación a la primera, se conformaron díadas integradas por un niño nivel 2 y otro nivel 3 (ambas respuestas erróneas pero diferentes); en relación a la segunda, 
se conformaron díadas integradas por un niño nivel 2 y otro nivel 4 (asimetría acentuada), o por un niño nivel 3 y otro nivel 4 (asimetría moderada). También se analizaron díadas netamente simétricas, es decir, integradas por dos niños de nivel 2 o por dos niños de nivel 3. La principal conclusión estuvo a favor de la asimetría de competencia epistémica: el avance cognitivo más notorio tiene lugar cuando un sujeto con una respuesta errónea interactúa con un compańero que ya ha construido la noción lógica en cuestión.

\section{Interacción sociocognitiva en la resolución de matrices lógicas}

En el presente trabajo se analiza la cuestión de la simetría/asimetría de competencia epistémica en un tipo de tarea diferente a los mencionados hasta el momento: la resolución de ítems lógicos en matrices progresivas. Esta decisión se inserta en una línea de trabajo que utilizó este formato de actividad como base para el análisis de la interacción sociocognitiva (Fernández, Wegeriff, Mercer \& Rojas-Drummond, 2001; Litteton et al., 2005; Mercer, Wegerif \& Dawes, 1999; RojasDrummond; Mazón, Fernández \& Weferif, 2006; Rojas-Drummond \& Mercer, 2003; Rojas-Drummond \& Peón Zapata, 2004; Roselli, 1986, 1988, 1999, 2004, 2010, 2011; Roselli, Bruno \& Evangelista, 2004a, 2004b; Roselli, Gimelli \& Hechen, 1995; Wegerif et al., 2016; Wegerif, Mercer \& Dawes, 1999; Webb \& Treagust, 2006; Yang, 2016). La tarea presenta un formato general común — más allá de la forma específica adoptada en cada estudio- consistente en una serie de problemas lógicos, en un orden de dificultad creciente. Cada problema o ítem está constituido por un conjunto de figuras (estímulos visuales) ordenados en filas y columnas, que guardan una relación de sentido lógico entre sí. En cada ítem se encuentra ausente la última figura que completa esta secuencia por filas y columnas. La resolución de cada problema implica deducir la relación de sentido lógico que une a las figuras y proponer, entre un conjunto de opciones de respuesta disponibles, la figura ausente que completa la secuencia. 
Originalmente (por ejemplo, Roselli, 1986, 1988), la utilización de esta tarea tuvo un sentido evolutivo: extender los estudios sobre interacción sociocognitiva centrados en el momento de transición entre no conservación y conservación (cinco y seis años aproximadamente) a un período del desarrollo cercano a la construcción de una lógica operatoria formal (diez y once años aproximadamente) y edades más avanzadas (por ejemplo, universitarios). Esto implica, a su vez, que la utilización de esta tarea se aleja de su propósito fundacional, que fue establecer psicométricamente el nivel de inteligencia general individual. A diferencia de ello, su aplicación simplemente apunta a la construcción de un dispositivo o situación que active procesos colectivos lógicos, con el fin de estudiar la interacción sociocognitiva, es decir, la resolución intersubjetiva de problemas.

Una parte de estos estudios (por ejemplo, Fernández et al., 2001; Mercer, et al., 1999; Rojas-Drummond et al., 2006; Rojas-Drummond \& Mercer, 2003; Rojas-Drummond \& Peón Zapata, 2004; Wegerif et al., 1999; Webb \& Treagust, 2006; Yang, 2015) trabajó con un tipo de adaptación del Test de Matrices Progresivas de Raven (Raven, 1991). La versión original, de 60 ítems, fue dividida en dos series paralelas de 30 ítems cada una (una serie integrada por los ítems pares y la otra por los impares). El diseńo básico consistió en utilizar ambas series de ítems para efectuar mediciones pretest-postest (en base a la sumatoria de problemas correctos): una de estas series estuvo referida a la performance individual (el individuo resolvía solitariamente los 30 ítems) y la otra estuvo referida a la performance colectiva (los compañeros resolvían conjuntamente los otros 30 ítems). Entre ambas instancias de evaluación (pretest y postest), los sujetos participaban de una experiencia de formación e incentivación del trabajo colaborativo (por ejemplo, el programa de intervención llamado Thinking Together).

En el caso puntual del pretest-postest colectivo, los autores propusieron tres formas básicas de construcción de la solución, tomando como principal indicador la calidad del intercambio lingüístico y la consiguiente posibilidad de lograr un auténtico entendimiento compartido sobre los significados relativos a la tarea: exploratoria, acumulativa 
y de disputa. La primera representa el nivel de máxima calidad en el intercambio lingüístico colectivo, ya que los participantes logran una auténtica construcción de significados compartidos a partir de la confrontación crítica de ideas. El diálogo se caracteriza por el ofrecimiento de sugerencias y alternativas de acción, ambas puestas a prueba por el grupo.

Por su parte, la línea de Roselli (Roselli, 1986, 1988, 1999, 2004, 2010; Roselli et al., 1995, 2004a, 2004b) presenta dos diferencias metodológicas básicas respecto al diseño anterior. Primero, trabajó con otro tipo de adaptaciones de la versión original de la prueba de matrices progresivas, construyendo series paralelas de menor cantidad de ítems, algunos seleccionados de la serie original y otros construidos ad-hoc. Segundo, una de las series se utilizó para una medición pretest-postest, únicamente de la performance individual; la otra se aplicó en una fase intermedia colaborativa, a partir de la cual se obtenía una medición de la performance colectiva y de la modalidad interactiva desplegada por los compańeros de trabajo (por ejemplo, la díada). Inicialmente, propusieron tres modalidades sociocognitivas en la resolución colaborativa de problemas, operacionalizadas a partir de dos indicadores estrictamente formales: la cantidad de interacciones (alta - baja) y la simetría del intercambio (alta - baja): a) sintética-integradora (alta interacción - alta simetría), justo equilibrio entre las performances individuales (proceso cooperativo en el sentido estricto de la palabra donde hay consenso interpersonal y consideración del punto de vista ajeno); b) coincidente-unilateral (alta interacción - baja simetría; baja interacción - alta simetría; media interacción - media simetría), la estrategia grupal se adscribe a la estrategia de uno de los miembros (relación asimétrica donde uno de los participantes influye y absorbe el papel del otro); c) incoherente-irregular (baja interacción - baja simetría), solo se producen transitorios acercamientos a cada performance individual, alternado bruscamente la secuencia grupal (cada miembro funciona de manera esquizoide en relación a los demás).

Posteriormente, Roselli (1999) modificó este sistema por una necesidad de superación del carácter formal de las modalidades 
identificadas, utilizando criterios de operacionalización alternativos relativos al contenido de las verbalizaciones, es decir, con significación cualitativa. A partir de dicha revisión diferenció dos modalidades principales: a) constructiva, donde la interacción consiste en un genuino trabajo en equipo, con alternancia en las intervenciones de los sujetos y coordinación de las diferentes perspectivas, con el objetivo de construir una solución compartida. Al interior de este modalidad se diferencian dos subcategorías: por un lado, puede ser plural y heterogénea, es decir, existe una verdadera confrontación de ideas y la posterior construcción colectiva de los conocimientos; por otro lado, también puede ser unidireccional ya que, si bien hay trabajo de equipo, los sujetos coinciden y permanecen en una única perspectiva, sin que ocurra una auténtica confrontación de puntos de vista diferentes; b) individual, en la cual la interacción se caracteriza por un predominio de la reflexión personal y la necesidad de negociar con el compañero una decisión compartida.

El fuerte énfasis en la propia postura de cada sujeto no afecta la posibilidad de lograr un acuerdo intersubjetivo, pero a diferencia de la modalidad constructiva el grado de implicación interpersonal no constituye un elemento definitorio. También aquí es necesario efectuar una discriminación en dos subcategorías: por un lado, puede ser egocéntrica, caracterizada por un mínimo intercambio y la respuesta pseudogrupal es negociada sin interés de convencer al otro mediante argumentaciones; por otro lado, puede ser instructiva o enseñante, en donde sí existe un deseo de comunicar y convencer en función de un argumento fundamentado. De esta manera, en ambas modalidades principales se pueden encontrar formas de colaboración, tanto avanzadas (constructiva plural e individual instructiva) como rudimentarias (constructiva unidireccional e individual egocéntrica).

\section{El presente estudio}

La presente investigación pretendió sintetizar ambas líneas que enfocan el estudio de la interacción sociocognitiva en tareas de resolución de matrices lógicas. Se propuso el análisis de la interacción 
sociocognitiva (y su relación con el avance individual) en ítems específicos, que reproducían la simetría o asimetría de competencia epistémica general. Esto implicó, en el marco de resolución de series lógicas (como las aquí planteadas), tener en cuenta dos aspectos complementarios en lo que concierne a los criterios de simetría y asimetría: a) la sumatoria de ítems resueltos correctamente por cada individuo en el pretest y la igualdad/desigualdad cuantitativa respecto a la su compañero (criterio general); b) en combinación con lo anterior, la complementación del criterio de igualdad o desigualdad genérica con un criterio referido a los problemas específicos que fueron resueltos de manera correcta o incorrecta por cada sujeto de la díada (como se detalla más adelante).

El objetivo general del trabajo fue analizar la relación entre interacción entre pares y avance cognitivo individual, en situaciones de simetría y asimetría de competencia epistémica. Puntualmente, la investigación persiguió tres propósitos específicos, siempre tomando como eje básico la comparación de diferentes formas de simetría y asimetría epistémica: (a) analizar el avance cognitivo individual, independientemente de la modalidad interactiva; (b) analizar la interacción diádica como proceso, esto es, el aporte y distribución de mensajes argumentales; (c) analizar el avance cognitivo individual en lo anterior (proceso interactivo).

\section{Método}

Se utilizó un diseño de tres momentos: pretest individual, fase colaborativa y postest individual. Cada sujeto resolvió individualmente una serie de diez ítems de dificultad creciente (pretest). A partir de esta evaluación, se determinó el nivel de competencia individual (baja o alta), en base a la sumatoria de los ítems resueltos correctamente. Sobre este criterio, más la diferencia relativa entre la propia puntuación y la de un potencial compañero de díada, cada sujeto participó en una de las tres formas de integración sociocognitiva analizadas en la fase colaborativa: simetría de dos niños de baja competencia $(n=10)$, simetría de dos niños de alta competencia $(n=15)$ y asimetría entre un niño de baja competencia y uno de alta competencia $(n=11)$. En esta fase 
interactiva, cada díada resolvió otra serie de diez ítems, paralela a la del pretest. Finalmente, el postest consistió en la resolución individual de la misma serie lógica del pretest.

\section{Participantes}

Participaron 72 sujetos con una edad media de 11 ańos, 3 meses ( $D E=7$ meses), de nivel socioeconómico medio, de quinto y sexto grado de nivel primario. Los estudiantes pertenecían a dos escuelas de Rosario (Argentina) con perfiles institucionales similares entre sí, en tanto ambas eran parte de las instituciones de gestión oficial (pública) con mayor historia y trayectoria en la ciudad, ubicadas en la zona céntrica de la misma. De la muestra disponible inicial se descartaron casos potenciales por no contar con algunos de los consentimientos correspondientes (del responsable del niño y/o del mismo) o estar ausente en alguna de las fases del estudio. La selección muestral no incluyó casos atípicos (por ejemplo, con trastornos en algún área del desarrollo).

\section{Medición}

Se utilizó una serie de diez ítems lógicos de dificultad creciente, directamente extraídos y/o adaptados del Test de Matrices Progresivas de Raven, Escala General (Raven, 1991). Cada ítem estaba constituido por un conjunto de figuras (estímulos visuales) ordenados en filas y columnas (tres figuras por fila por tres figuras por columna) que guardaban una relación de sentido lógico entre sí. La última figura que completaba la secuencia estaba ausente. La resolución de cada problema implicaba deducir la relación de sentido lógico que unía a las figuras y proponer, entre un conjunto de ocho opciones de respuesta disponibles, cuál correspondía a la figura correcta.

En este caso se utilizaron dos versiones paralelas (A y B) de la serie de diez ítems lógicos, ambas construidas ad hoc. La serie A se aplicó en el pretest y en el postest individuales, mientras que la serie B en la sesión interactiva. El paralelismo entre ambas versiones concernió tanto a la estructura lógica de los ítems como al comportamiento resolutorio 
de los mismos. Esto último fue determinado a partir de una prueba piloto anterior al estudio propiamente dicho, con una muestra de nińos de cursos similares a los indagados aquí, que resolvieron dos series con mayor número de ítems a los aplicados en este trabajo (con contrabalanceo del orden de administración). A partir de esta medición, se constituyeron dos versiones definitivas integradas por diez pares de ítems que no habían registrado diferencias significativas entre sí en cuanto al grado de acierto/error (acierto=1, error=0; prueba $T$ de Student para muestras relacionadas, $p=n$.s.). Este paralelismo entre ambas versiones no solo aportó rigurosidad al diseńo (al establecer niveles de dificultad constantes en las tres fases del estudio), sino que también garantizó la confiabilidad de la propia serie a partir de la comparación entre dos formatos equivalentes o alternativos.

Tal como se mencionó al principio, la utilización de esta tarea en el presente estudio se alejó de su propósito fundacional, que fue establecer psicométricamente un nivel de inteligencia general individual. A diferencia de ello, su aplicación simplemente apuntó a la construcción de un dispositivo de activación de procesos colectivos lógicos, con el fin de estudiar la interacción sociocognitiva, es decir, la resolución intersubjetiva de problemas. Aún así, la construcción de la serie y el ordenamiento de sus ítems se inspiró en las series de la prueba original, en tanto la determinación de la dificultad de los ítems y su consiguiente ordenamiento progresivo se decidieron a partir de la proporción (de mayor a menor) de sujetos de la muestra piloto que había resuelto exitosamente cada problema.

Además de la serie lógica propiamente dicha (integrada por diez ítems), se propusieron otros dos ítems de baja dificultad en una fase de ensayo, que los sujetos resolvieron previamente para asegurar una correcta comprensión de la consigna y una familiarización con la tarea.

\section{Procedimiento}

El proyecto estuvo avalado por el Tribunal de Ética correspondiente. Se solicitaron los respectivos consentimientos informados de las auto- 
ridades de cada escuela, los padres de los alumnos y los propios niños. No participaron del estudio aquellos alumnos que no contaron con el permiso de sus padres y/o que no quisieron hacerlo por propia voluntad.

Antes del pretest individual cada sujeto realizó los dos ítems adicionales de ensayo. El pretest consistió en la resolución individual de la serie lógica de diez ítems de dificultad creciente, lo cual arrojó un puntaje total entre 0 y 10 (recuento de aciertos). La distribución general de dichos puntajes fue dividida en dos partes, en función del valor de mediana. Consecuentemente, los niños con puntajes 0-5 fueron considerados de baja competencia, mientras aquellos con puntajes 6-10 fueron considerados de alta competencia. Esta evaluación inicial se utilizó como un criterio para la conformación de las diferentes condiciones de comparación. Hubo tres tipos de díadas: simetría bajo-bajo, simetría alto-alto y asimetría bajo-alto. Para la conformación de las díadas no solo se consideró el nivel general de cada sujeto $(\leq 5 \mathrm{o}>5)$, sino también la diferencia relativa entre los puntajes individuales (en simetría: $\leq 2$ puntos; en asimetría: $\geq 4$ puntos).

Posteriormente, una vez constituidas las díadas, estas resolvieron colaborativamente la otra serie de diez problemas, paralela a la del pretest. Esto se realizó entre una y tres semanas posteriores al pretest. La consigna indicaba a los integrantes de la díada trabajar conjuntamente, discutir posibles soluciones de cada problema y proponer una respuesta colectiva final (incluso aunque haya persistido el desacuerdo entre ambos). En todas las díadas se audio-registró y transcribió la interacción sociocognitiva. Finalmente el postest, realizado inmediatamente después de la fase intermedia, planteaba la resolución individual de la misma serie lógica del pretest.

\section{Análisis de los datos}

(a) Análisis del avance cognitivo individual (independientemente de la interacción)

En primer lugar, se analizó el avance individual general, es decir, la diferencia entre los totales (sobre la serie general de diez ítems) 
comparando los resultados del pretest con los del postest. Esta comparación de medias pretest-postest fue entre las condiciones colaborativas analizadas. Se aplicó análisis de varianza de medidas repetidas, puesto que se consideró un factor intersujeto (condición colaborativa) y un factor intrasujeto (pretest-postest), en relación a la variable dependiente avance cognitivo individual (con distribución normal).

En segundo lugar, también se consideró el avance individual referido a los ítems específicos de simetría o asimetría de competencia epistémica. En función de este criterio, el progreso pretest-postest de cada individuo, en cualquier nivel de competencia, se analizó en relación a dos ítems específicos tomados como referencia diádica. En el caso de las díadas conformadas por sujetos igualmente deficitarios, dentro de la baja competencia, se analizaron dos ítems que en el pretest habían recibido respuestas incorrectas. Estos eran de dificultad media para que correspondieran con la propia ZDP, pudiendo ser el ítem número 5, 6 y/o 8 de la serie. El mismo criterio se adoptó en relación a cada individuo de alta competencia que trabajó con un compañero de similar condición, pero los dos ítems de referencia podían ser el 8, 9 y/o 10 (obviamente, de mayor dificultad que el 5 y 6 utilizados con la condición simétrica de baja competencia). Finalmente, para los individuos de baja competencia que trabajaron con un compañero de alta competencia (asimetría) también se tomó como referencia dos de los ítems 5, 6 y/o 8 (aunque aquí se excluyó del análisis al compañero de alta competencia, que en el pretest había resuelto correctamente esos problemas).

Considerando que el análisis referido a ítems específicos siempre se basó en dos ítems resueltos de modo incorrecto en el pretest, el incremento pretest-postest (variable dependiente) se determinó directamente por la resolución correcta o no de esos mismos dos ítems en el postest. El valor posible de postest fue 0,1 o 2, según la cantidad de ítems resueltos correctamente. Para la comparación de las condiciones se utilizó análisis de varianza de un factor, donde se analizó un factor intersujeto (condición colaborativa) en relación a la variable dependiente diferencia pretest-postest (con asunción de normalidad). De los 
sujetos medidos inicialmente en el pretest solo se consideraron aquéllos que cumplían los requisitos referidos, lo que supuso omitir algunos casos de aquí en adelante.

\section{(b) Análisis del proceso interactivo diádico en items específicos}

La interacción diádica se analizó en relación a dos aspectos: volumen de la argumentación de la díada y distribución del mismo entre ambos compañeros. Se utilizó la definición de Toulmin (2003), quien entiende al argumento como un intento de justificación, es decir, el sujeto expone una idea sobre un aspecto del problema cuya validez se mide por la coherencia entre la misma y la información que se utiliza para justificarla.

Por cada díada se calculó el volumen general de argumentación, dividiendo la cantidad de mensajes argumentales por el total general de mensajes. Por su parte, la distribución del aporte de mensajes argumentales entre los compańeros de díada se calculó dividiendo el número de mensajes aportados por cada participante. En esta división, el valor del numerador correspondió al sujeto con menor aporte de mensajes, de manera de arrojar un valor entre 0 (asimetría absoluta) y 1 (simetría absoluta).

En este apartado, el análisis del proceso interactivo (puntualmente, las variables de volumen y distribución argumental de la díada) se realizó en dos sentidos. Primero, en los dos ítems específicos correspondientes a la propia ZDP se compararon las díadas simétricas del sector de baja competencia general con las del sector de alta competencia general. Segundo, el mismo análisis comparativo se realizó entre díadas simétricas del sector de baja competencia y las díadas asimétricas (sector mixto de baja/alta competencia), pero tomando como referencia los mismos ítems. En ambos casos, se aplicó prueba “ $T$ ” de Student (muestras independientes) para comparar las medias de las variables procesuales entre las condiciones referidas. 
(c) Análisis de la relación entre el proceso interactivo y el avance cognitivo individual en items especificos

En este apartado se reprodujo la secuencia analítica del punto (b), pero considerando como variable dependiente única al incremento pretest-postest en ítems específicos. Por su parte, la composición de la díada según competencia epistémica y las dos variables procesuales de la interacción fueron consideradas alternadamente como variables independientes en los diferentes análisis de varianza realizados. Las dos variables relativas al proceso interactivo (volumen y distribución argumentales), expresadas originalmente en valores continuos, fueron dicotomizadas para considerarlas como posibles factores en los análisis de varianza implementados. El volumen de argumentación de la díada se dividió en dos categorías sobre el valor de mediana de la distribución de valores observados sobre el total general $(M d n=.15)$ : baja argumentación $(\leq .15)$ y alta argumentación $(>0.15)$. La distribución del aporte argumental también se dicotomizó: asimetría en el intercambio argumental $(<.50)$ y simetría en el intercambio argumental $(\geq .50)$.

\section{Resultados}

\section{El avance cognitivo individual (independientemente de la interacción)}

Se registró un avance significativo pretest-postest en todas las condiciones $[F(1,68)=5.71, p<.05]$. Pero, además, se observó una interacción significativa entre condición colaborativa y avance pretestpostest $[F(3,68)=3.35, p<.05]$. La tabla 1 muestra que los avances más marcados se dieron en los niños de baja competencia que trabajaron con un compañero más competente (dif. pretest-postest = +1.75 ), seguidos por niños de baja competencia que trabajaron con otro de similar condición (dif. pretest-postest $=+.75$ ). En cambio, los sujetos de alta competencia no mostraron avances (incluso retrocesos mínimos), independientemente del grado de competencia del compañero (bajo o alto). 


\section{Tabla 1}

Valores pretest-postest individuales generales

\begin{tabular}{lcccc}
\hline & \multicolumn{2}{c}{ Pretest } & \multicolumn{2}{c}{ Postest } \\
\cline { 2 - 5 } & $M$ & $D E$ & $\mathrm{M}$ & $\mathrm{DE}$ \\
\hline Bajo con bajo & 4.60 & .99 & 5.35 & 1.39 \\
Alto con alto & 6.83 & .87 & 6.77 & 1.59 \\
Bajo con alto & 3.91 & .94 & 5.18 & 1.08 \\
Alto con bajo & 8.18 & .87 & 8.00 & 1.67 \\
\hline
\end{tabular}

En relación al análisis centrado en los ítems específicos (propios de la ZDP de cada nivel de competencia individual), la comparación de los avances individuales entre las diferentes condiciones no arrojó diferencias significativas, aunque la mayor diferencia entre medias pretest-postest se dio en los individuos de nivel bajo que trabajaron con un compañero de alta competencia $(M=.70 ; D E=.67)$, seguidos por los niños de nivel bajo que trabajaron con un compañero de similar condición $(M=.39 ; D E=.61)$ y por último los sujetos de alta competencia que trabajaron con un par $(M=.33 ; D E=.48)$.

\section{El proceso interactivo diádico en ítems específicos}

La comparación entre las díadas simétricas de baja y de alta competencia, en ítems específicos, mostró diferencias significativas en relación a la distribución del aporte argumental $[T(30)=-3.12, p<.01]$. Las díadas simétricas de alta competencia registraron patrones de mayor simetría en el aporte argumental $(M=.52 ; D E=.30)$ que las simétricas de baja competencia $(M=.17 ; D E=-32)$. En cambio, el volumen general de argumentación no mostró diferencias significativas entre ambas condiciones $(M=.19 ; D E=.12$ y $M=.16 ; D E=.13$, respectivamente).

Por su parte, la comparación entre las díadas simétricas de baja competencia y las díadas asimétricas, al interior de los ítems específicos, no arrojó diferencias significativas en ambas variables procesuales (volumen de argumentación, $M=.19 ; D E=.12$ y $M=.18 ; D E=.11$, 
respectivamente; distribución del aporte argumental, $M=.17 ; D E=.32$ y $M=.21 ; D E=.32$, respectivamente).

\section{Relación entre el proceso interactivo y el avance cognitivo individual en items especificos}

Primero, se analizó el avance cognitivo individual en ítems específicos correspondientes a la propia ZPD, en función de la condición colaborativa simétrica (bajo-bajo o alto-alto) y el volumen de argumentación de la díada (baja o alta). El volumen de argumentación mostró un efecto principal significativo sobre el progreso individual $[F(1,35)$ $=4.21, p<.05]$, independientemente del sector de competencia (bajo o alto). Los sujetos que participaron en interacciones con alto volumen argumental mostraron los mayores incrementos pretest-postest individuales. Incluso, cuando la díada de alto volumen argumentativo correspondió a dos sujetos del sector de competencia alta, esta tendencia se reforzó aunque sin lograr significatividad estadística (ver valores en la tabla 2).

\section{Tabla 2}

Avance cognitivo individual en items correspondientes a la propia ZDP, según simetría (baja y alta), volumen de argumentación de la diada y distribución del aporte argumental

\begin{tabular}{|c|c|c|c|c|c|c|c|c|}
\hline & \multicolumn{8}{|c|}{ Diferencia pretest-postest } \\
\hline & \multicolumn{4}{|c|}{$\begin{array}{l}\text { Argumentación de } \\
\text { la díada }\end{array}$} & \multicolumn{4}{|c|}{$\begin{array}{l}\text { Distribución del aporte } \\
\text { argumental }\end{array}$} \\
\hline & \multicolumn{2}{|c|}{ Baja } & \multicolumn{2}{|c|}{ Alta } & \multicolumn{2}{|c|}{ Equitativa } & \multicolumn{2}{|c|}{ Desigual } \\
\hline & $M$ & $D E$ & $\mathrm{M}$ & $\mathrm{DE}$ & M & $\mathrm{DE}$ & M & $\mathrm{DE}$ \\
\hline Bajo con bajo & .25 & .46 & .50 & .71 & .00 & .00 & .50 & .65 \\
\hline Alto con alto & .17 & .39 & .67 & .52 & .30 & .48 & .75 & .50 \\
\hline
\end{tabular}


Luego se realizó un análisis similar al anterior, aunque variando uno de los factores. Puntualmente, el avance pretest-postest se consideró en función de la condición colaborativa simétrica (bajo-bajo o alto-alto) y la distribución del aporte argumental (equitativo o desigual). Se detectó un efecto principal significativo del segundo $[F(1$, $31)=4.51, p<.05]$, ya que los sujetos que participaron en interacciones con una distribución desigual del aporte argumental, concentrando el mayor volumen, fueron los que registraron mayores incrementos pretest-postest. No se observó un efecto de interacción significativo entre ambos factores.

La comparación entre sujetos de nivel bajo que trabajaron con un par (simetría de baja competencia) y sujetos de nivel bajo que trabajaron con uno de mayor competencia (asimetría), en combinación con las variables procesuales, no mostró diferencias significativas en relación al incremento pretest-postest en ítems específicos. Si bien una mayor argumentación se vinculó con mayores avances individuales, esto no se dio de manera diferencial en el grado de competencia del compañero (baja o alta) de niños de baja competencia.

\section{Discusión}

La presente investigación propuso analizar el proceso de interacción sociocognitiva y su efecto de avance individual en situaciones de simetría y asimetría de competencia epistémica. La competencia individual, así como el grado de similitud o diferencia de esta respecto a la del compañero de díada, fue considerada desde dos criterios complementarios: general (basado en el puntaje total de aciertos en la serie de problemas lógicos del pretest) y específico (basado en la capacidad de resolución de ítems específicos que componían esa serie inicial). En función de una combinación entre ambos criterios, se propusieron tres objetivos: (a) analizar el avance cognitivo individual, sin considerar la interacción; (b) analizar el proceso interactivo diádico; (c) analizar la relación entre proceso interactivo diádico y avance cognitivo individual. 
El objetivo (a), en el que se comparó el avance cognitivo de las diferentes integraciones diádicas en función de la competencia (bajo-bajo, bajo-alto, alto-bajo, alto-alto), tuvo un fin básicamente preliminar o exploratorio porque no consideró ni el proceso interactivo, ni tampoco este mismo proceso al interior de ítems específicos (que reproducían el nivel de competencia general inicial), ambos aspectos vinculados directamente con el objetivo central del estudio. Desde una perspectiva interaccionista de la inteligencia (Mugny \& Pérez, 1988; Psaltis et al., 2009) resulta imprescindible incorporar el análisis de diferentes características de la interacción colaborativa (en este caso, el volumen y distribución del aporte argumental) para comprender de manera cabal la relación entre avance cognitivo individual y composición sociocognitiva de la díada. Los resultados de este primer análisis exploratorio son coherentes con otros estudios previos que se limitaron exclusivamente a comparar pretest-postest sin incluir la interacción de la díada (por ejemplo, Asterhan et al., 2014, o Fawcett \& Garton, 2005), pero aquí se enfatiza la necesidad de superar esta perspectiva, incluyendo al proceso sociocognitivo como requisito ineludible para lograr una comprensión más integral del fenómeno, tal como se propuso en los objetivos (b) y (c).

Estos objetivos, que hicieron al núcleo central del artículo, incluyeron el estudio de la interacción al interior de ítems específicos, atendiendo especialmente a dos aspectos: el volumen de argumentación en la díada y la distribución del aporte argumental entre los compañeros de trabajo. El estudio de la relación entre composición sociocognitiva de la díada y argumentación (sin entrar a considerar el avance cognitivo individual derivado), en ítems específicos correspondientes a la propia ZDP, sugiere —en término globales— una asociación entre un nivel alto de competencia en ambos integrantes de la díada (simetría de alta competencia) y una distribución equitativa del aporte argumental. En otras palabras, el nivel de competencia se asocia con la posibilidad de establecer interacciones y regulaciones más equitativas, convocando a una mayor comunalidad cognitiva, lo cual se lograría a menor escala (o incluso no se encontraría) en díadas de sujetos de baja 
competencia epistémica o con asimetría de competencia. Este hallazgo se corresponde con la relación teórica clásica entre desarrollo cognitivo y coordinación social propuesta de Piaget (1964/1995), quien entiende a esta última como manifestación de la evolución de las estructuras lógicas del sujeto.

En una línea similar, aunque desde una revisión crítica de la teoría piagetiana, la Escuela de Ginebra ha propuesto el concepto de prerrequisito cognitivo como condición básica para el intercambio sociocognitivo auténtico (Gilly, 1992), más allá de revalorizar el intercambio entre sujetos de similar nivel de competencia general. Por tanto, siguiendo a Peralta y Roselli (2016) y a Roselli (1999), podría pensarse que las dificultades para establecer aportes argumentales equitativos por parte de los sujetos de baja competencia se vinculan con la ausencia o escaso desarrollo de dichos esquemas elementales en los casos de menor desarrollo de la función.

Al mismo tiempo, esta distribución desigual del aporte argumental en las díadas simétricas de baja competencia también explicaría la ausencia de diferencias significativas de estas con respecto a las díadas asimétricas, las cuales — acorde a las expectativas iniciales - también mostraron patrones desparejos de intercambio argumental. Sin embargo, es probable que en cada caso dichas distribuciones argumentales no equitativas encuentren diferentes explicaciones. Esto es, en las díadas con asimetría de competencia epistémica la desigualdad del intercambio argumental estaría vinculada principalmente a un elemento de liderazgo cognitivo del sujeto más competente; es decir, es esperable que la mayoría de las argumentaciones provengan principalmente del mismo. En cambio, en las díadas simétricas de baja competencia la distribución desigual de las argumentaciones se explicaría por la dificultad para establecer regulaciones equitativas que aseguren una comunalidad cognitiva, producto de un menor desarrollo de la sociabilidad simétrica asociada a la baja competencia cognitiva general, como ya se mencionó previamente. Además, siguiendo esta lógica, es esperable que la interacción asuma modalidades básicamente relacionales (en lugar de epistémicas), o sea, que los procesos de influencia social se definan 
principalmente por cuestiones de posición social más que por justificaciones racionales o argumentos (Quiamzade, Mugny \& Darnon, 2009).

La consideración de estos elementos no cognitivos o psicosociales constituye una dimensión cada vez más presente en las investigaciones sobre interacción entre pares y desarrollo cognitivo. En otras palabras, ante niveles bajos de competencia específica en los compañeros de trabajo, es esperable que la modalidad del intercambio y los procesos de influencia social se definan prioritariamente por aspectos psicosociales (no propiamente cognitivos), como puede ser la ascendencia (o dominancia), la sensibilidad social (Garton y Harvey, 2006), entre otros. En este sentido, podrían proponerse nuevas investigaciones centradas en estos aspectos no cognitivos de la interacción. Ello permitiría la superación de una visión epistémica clásica del hecho sociocognitivo por parte de una perspectiva alternativa de corte psicosocial que revalorice a la interacción como un proceso de influencia social e, incluso, atravesado por las representaciones recíprocas de los compañeros de trabajo (Quianzade, Mugny y Butera, 2014; Sorsana y Trognon, 2011).

Por otra parte, en el objetivo (c) se consideró la relación entre proceso interactivo y avance cognitivo individual en las diferentes situaciones de simetría y asimetría. En la comparación entre díadas simétricas de baja competencia con las de alta competencia, el volumen de argumentación de la díada y su distribución equitativa constituirían factores claves para el avance cognitivo individual. Al mismo tiempo, aún sin alcanzar niveles de significatividad estadística, la influencia del volumen de la argumentación como predictor de avance cognitivo individual sería mayor en las díadas simétricas de alta competencia. Este dato condice con los antecedentes que rescatan el valor de una modalidad epistémica por sobre una modalidad puramente relacional. Esta modalidad epistémica está basada en la confrontación equitativa entre posiciones individuales en base a argumentaciones y justificaciones lógicas y la búsqueda de un consenso cognitivo (por ejemplo, Buchs et al., 2004; Peralta \& Roselli, 2016; Peralta, et al., 2012; Quiamzade \& Mugny, 2001). 
En síntesis, pueden proponerse dos conclusiones centrales. Primero, la principal diferenciación entre las condiciones diádicas analizadas (simetría de baja competencia, simetría de alta competencia y asimetría) se da en relación al propio proceso interactivo, puntualmente, en relación a la distribución del aporte argumental (no al volumen de argumentación). En este sentido, pueden diferenciarse tres niveles de equidad y comunalidad del intercambio, cada uno asociado a una composición diádica particular: simetría de alta competencia (distribución igualitaria de argumentos, con retroalimentación), asimetría (distribución desigual de los argumentos, producto de la desigualdad cognitiva entre los sujetos) y simetría de baja competencia (distribución desigual de los argumentos, producto del bajo nivel de desarrollo social simétrico de los sujetos). Segundo, en cuanto a la relación entre proceso interactivo y avance cognitivo individual, las variables procesuales (volumen y distribución de la argumentación) serían los factores asociados al avance cognitivo individual, más allá de la condición diádica de simetría o asimetría (aunque podría proponerse que esta tendencia hacía un mayor avance cognitivo individual se daría especialmente en las díadas simétricas de alta competencia).

Las conclusiones previas tienen implicancias prácticas directas en relación al ámbito escolar y la planificación de estrategias didácticas basadas en el aprendizaje colaborativo, aunque con cierta cautela considerando que esta investigación centró su análisis en unidades sociales mínimas (díadas) y en una tarea de resolución de problemas no propiamente escolar. Por un lado, la conformación de diferentes colectivos de trabajo, según los niveles de competencia específica de sus integrantes, supondría para el docente el seguimiento de diferentes modalidades de intercambio sociocognitivo entre los estudiantes. Por el otro, queda claro que la simple conformación de equipos de trabajo no es sinónimo de aprendizaje, sino que es fundamental la presencia y distribución equitativa de argumentaciones entre los compañeros. Así, se torna necesario la formación de los alumnos y de los propios docentes en competencias argumentativas (Santibañez, 2016), las cuales constituyen una vía privilegiada para la negociación de significados y la 
construcción de una auténtica intersubjetividad (Rogoff, 2012). Considerando estas implicancias prácticas del trabajo, y tratando de superar una posición puramente especulativa, sería pertinente extender este tipo de investigaciones a tareas que involucren el aprendizaje de conocimientos escolares específicos y en colectivos más numerosos que díadas (por ejemplo, tríadas o tétradas).

Para finalizar, vale tener en cuenta algunas limitaciones del presente trabajo. Una de ellas estuvo referida a que, en algunos casos, se establecieron comparaciones entre condiciones diádicas sobre ítems diferentes entre sí. Por ejemplo, el análisis de ítems propios de la ZDP de díadas simétricas de diferentes niveles de competencia (bajo o alto), implicó necesariamente trabajar con ítems diversos, acordes a cada condición. Desde un punto de vista metodológico ello implicó la desventaja de establecer comparaciones sobre condiciones no específicamente equivalentes, si bien esta situación obedeció a un fundamento teórico-metodológico necesario.

Otra limitación del estudio (que ya es parte inherente de nuestros abordajes) es el tamaño de la muestra y la dificultad de asegurar la misma cantidad de integrantes entres las distintas condiciones consideradas, lo cual dificulta una generalización estadística rigurosa. Sin embargo, debe recordarse que ello constituye un costo necesario, vinculado con la factibilidad del estudio, especialmente si se tiene en cuenta la intención de adentrarse en los procesos interactivos involucrados.

\section{Referencias}

Asterhan, C., Schwarz, B. \& Cohen-Eliyahu, N. (2014). Outcome feedback during collaborative learning: Contingencies between feedback and dyad composition. Learning and Instruction, 34, 1-10. https://doi.org/10.1016/j.learninstruc.2014.07.003

Buchs, C., Butera, F., Mugny, G. \& Darnon, C. (2004). Conflict elaboration and cognitive outcomes. Theory Into Practice, 43(1), 23-30. https://doi.org/10.1207/s15430421 tip4301_4 
Denessen, E., Veenman, S., Dobbelsteen, J. \& Van Schilt, J. (2008). Dyad composition effects on cognitive elaboration and student achievement. Journal of Experimental Education, 76(4), 363383. https://doi.org/10.3200/JEXE.76.4.363-386

Doise, W. \& Mugny, G. (1984). The social development of the intellect. Oxford: Pergamon.

Fawcett, L. \& Garton, A. (2005). The effect of peer collaboration on children's problem-solving ability. British Journal of Educational Psychology, 75(2), 157-169. https://doi. org/10.1348/000709904X23411

Fernández, M., Wegeriff, R., Mercer, N. \& Rojas-Drummond, S. (2001). Reconceptualizing "scaffolding" and the zone of proximal development in the context of symmetrical collaborative learning. Journal of Classroom Interaction, 36(2), 40-54.

Gabriele, A. J. (2007). The influence of achievement goals on the constructive activity of low achievers during collaborative problem solving. British Journal of Educational Psychology, 77(1), 121141. https://doi.org/10.1348/000709905X89490

Garton, A. \& Harvey, R. (2006). Does social sensitivity influence collaborative problem solving in children? A preliminary investigation. The Australian Educational and Developmental Psychologist, 23(2), 5-16. https://doi.org/10.1017/S0816512200028959

Garton, A. \& Pratt, C. (2001). Peer assistance in children`s problem solving. British Journal of Developmental Psychology, 19, 307318. https://doi.org/10.1348/026151001166092

Gilly, M. (1992). Introducción: Interacciones entre pares y construcciones cognitivas: modelos explicativos. En A.N. Perret-Clermont y M. Nicolet (Comps.), Interactuar y Conocer. Desafios y regulaciones sociales en el desarrollo cognitivo (pp. 23-31). Buenos Aires: Miño y Dávila.

Iiskala, T., Vauras, M. \& Lehtinen, E. (2004). Socially-shared metacognition in peer learning? Hellenic Journal of Psychology, 1, 147-178.

Iiskala, T., Vauras, M., Lehtinen, E. \& Salonen, P. (2011). Socially shared metacognition of dyads of pupils in collaborative mathe- 
matical problem-solving processes. Learning and Instruction, 21, 379-393. https://doi.org/10.1016/j.learninstruc.2010.05.002

Leman, P. \& Duveen, G, (1999). Representations of authority and children's moral reasoning. European Journal of Social Psychology, 29, 557-575. https://doi.org/10.1002/(SICI)10990992(199908/09)29:5/6<557::AID-EJSP946>3.0.CO;2-T

Leman, P. \& Duveen, G, (2003). Gender identity, social influence and children`s conversations. Swiss Journal of Psychology, 62(3), 149158. https://doi.org/10.1024//1421-0185.62.3.149

Light, P., Littleton, K., Messer, D. \& Joiner, R. (1994). Social and communicative processes in computer-based problem solving. European Journal of Psychology of Education, 9(2), 93-109. https://doi.org/10.1007/BF03173545

Littleton, K., Mercer, N., Dawes, L., Wegeriff, R, Rowe, D. \& Sans, C. (2005). Taking and thinking together at Key Stage 1. Early years, 25(2), 167-182. https://doi.org/10.1080/09575140500128129

Mercer, N., Wegerif, R. \& Dawes, L. (1999). Children's talk and development of reasoning in the classroom. British Education Research Journal, 25(1), 95-111. https://doi. org/10.1080/0141192990250107

Mugny, G. \& Pérez, J. (1988) Introducción de los editores. En G. Mugny y J. Pérez (Eds.), Psicología social del desarrollo cognitivo (pp. 17-44). Barcelona: Anthropos.

Peralta, N. \& Roselli, N. (2016). Conflicto sociocognitivo e intersubjetividad: análisis de las interacciones verbales en situaciones de aprendizaje colaborativo. Psicología, Conocimiento y Sociedad, 6, 90-113.

Peralta, N., Roselli, N. \& Borgobello, A. (2012). El conflicto sociocognitivo como instrument de aprendizaje en contextos colaborativos. Revista Interdisciplinaria, 29(2), 325-338.

Perret Clermont, A. (1980). Social interaction and cognitive development in children. London: Academic Press.

Piaget, J. (1995). Seis estudios de Psicología. Barcelona, España: Labor. (Año de publicación del original: 1964). 
Piaget, J. (2002). The language and thought of the child. London, England: Routledge (Original work published in 1923).

Psaltis, C. (2011). The constructive role of gender asymmetry in social interaction: Further evidence. British Journal of Developmental Psychology, 29, 305-312. https://doi. org/10.1111/j.2044-835X.2011.02029.x

Psaltis, C. \& Duveen, G. (2006). Social relations and cognitive development: The influence of conversation type and representations of gender. European Journal of Social Psychology, 36, 407-430. https://doi.org/10.1002/ejsp.308

Psaltis, C. \& Duveen, G. (2007). Conservation and conversation types: Forms of recognition and cognitive development. British Journal of Developmental Psychology, 25, 79-102. https://doi. org/10.1348/026151005X91415

Psaltis, C., Duveen, G. \& Perret Clermont, A. (2009). The Social and the Psychological: Structure and context in intellectual development. Human Development, 52(5), 291-312. https://doi. org/10.1159/000233261

Quiamzade, A. \& Mugny, G. (2001). Social influence dinamycs in aptitude tasks. Social Psychology of Education, 4, 311-334. https:// doi.org/10.1023/A:1011388821962

Quiamzade, A., Mugny, G. \& Butera, F. (2014). Psychologie sociale de la connaissance. Presses universitaires de Grenoble: Grenoble.

Quiamzade, A., Mugny, G \& Darnon, C. (2009). The coordination of problem solving strategies: When low competence sources exert more influence on task processing than high competence sources. British Journal of Social Psychology, 48, 159-182. https:// doi.org/10.1348/014466608X311721

Raven, J. (1991). Test de matrices progresivas para la medida de la capacidad intelectual (de sujetos de 12 a 65 años). Manual. Buenos Aires, Argentina: Paidós.

Rogoff, B. (2012). Learning without lessons: Opportunities to expand knowledge. Infancia y Aprendizaje, Journal for the Study of Education and Development, 35(2), 233-252. https://doi. org/10.1174/021037012800217970 
Rojas-Drummond, S. \& Mercer, N. (2003). Scaffolding the development of effective collaboration and learning. International Journal of Educational Research, 39(1-2), 99-111. https://doi. org/10.1016/S0883-0355(03)00075-2

Rojas-Drummond, S. \& Peón, M. (2004). Exploratory talk, argumentation and reasoning in Mexican primary school children. Language and Education, 18(6), 539-557. https://doi. org/10.1080/09500780408666900

Rojas-Drummond, S., Mazón, N., Fernández, M. \& Wegerif, R. (2006). Explicit reasoning, creativity and co-construction in primary school children's collaborative activities. Journal of Thinking Skills and Creativity, 1(2), 84-94. https://doi.org/10.1016/j. tsc.2006.06.001

Roselli, N. (1986). Análisis comparativo del proceso de elaboración cognitiva individual y grupal, en sujetos adolescentes, en relación a una tarea de resolución de problema. Rosario, Argentina: IRICE.

Roselli, N. (1988). Interacción social y desarrollo del pensamiento formal. En G. Mugny y J. Pérez (Eds.), Psicología Social del Desarrollo Cognitivo (pp. 211-224). Barcelona, España: Anthropos.

Roselli, N. (1999). La construcción sociocognitiva entre iguales. Fundamentos psicológicos del aprendizaje cooperativo. Rosario, Argentina: Ediciones IRICE.

Roselli, N. (2004). Resolución cooperativa de problemas en forma presencial y a distancia tipo chat en díadas y tétradas. Interdisciplinaria, 21(1), 71-97.

Roselli, N. (2010). Comparación experimental entre tres modalidades de enseñanza mediadas informáticamente. Revista de Investigación Educativa, 28(2), 265-282.

Roselli, N. (2011). Proceso de construcción colaborativa a través del chat según el tipo de tarea. Revista de Psicología, 29(1), 3-36.

Roselli, N., Bruno, M. \& Evangelista, L. (2004a). El trabajo cognitivo en equipo en forma presencial y mediada tipo chat en grupos de cuatro sujetos. Revista de Psicología General y Aplicada, 57(1), 95-111. 
Roselli, N., Bruno, M. \& Evangelista, L. (2004b). El chateo y la interacción social directa en el aprendizaje cooperativo de díadas. Revista Latinoamericana de Psicología, 36(3), 391-408.

Roselli, N., Gimelli, L. \& Hechen, M. (1995). Modalidades de interacción sociocognitiva en el aprendizaje de conocimientos en parejas. En P. Fernández Berrocal y M. A. Melero Zabal (Comps.), La interacción social en contextos educativos (pp. 137165). Madrid: Siglo XXI.

Roselli, N., Gimelli, L. \& Hechen, M. (1995). Modalidades de interacción sociocognitiva en el aprendizaje de conocimientos en parejas. En P. Fernández Berrocal y M. A. Melero Zabal (Comps.), La interacción social en contextos educativos (pp. 137165). Madrid, Espańa: Siglo XXI.

Santibáńez, C. (2016). Ecología Argumentativa Universitaria: Desde la Realidad a los Conceptos. Concepción: Cosmigonon Ediciones.

Sorsana, C. \& Trognon, A. (2011). Contextual determination of human thinking: About some conceptual and methodological obstacles in psychology studies. Human Development,54, 204233. https://doi.org/10.1159/000329844

Schmitz, M. \& Winskel, H. (2008). Towards effective partnerships in a collaborative problem-solving task. British Journal of Educational Psychology, 78, 581-596. https://doi. org/10.1348/000709908X281619

Toulmin, S. (2003). The Uses of Argument. Cambridge, UK: Cambridge University. https://doi.org/10.1017/CBO9780511840005

Tudge, J. (1992). Processes and consequences of peer collaboration: a vygotskian analysis. Child Development, 63, 1364-1379. https:// doi.org/10.2307/1131562

Tudge, J. \& Winterhoff, P. (1993). Can young children Benedit from collaborative problem solving? Tracing the effects of partner competente and feedback. Social Development, 2, 242-259. https://doi.org/10.1111/j.1467-9507.1993.tb00016.x

Tudge, J., Winterhoff, P. \& Hogan, D. (1996). The cognitive consequences of collaboration and feedback. Child Development, 67, 2892-2909. https://doi.org/10.2307/1131758 
Webb, P. \& Treagust, D. (2006). Using exploratory talk to enhance problem-solving and reasoning skills in grade-7 science classrooms. Research in Science Education, 36(4), 381-401. https:// doi.org/10.1007/s11165-005-9011-4

Wegerif, R., Fujita, T., Doney, J., Pérez Linares, J., Andrews, R. \& Rhyn, C. Developing and trialing a measure of group thinking. Learning and Instruction, 48, 40-50. https://doi.org/10.1016/j. learninstruc.2016.08.001

Wegerif, R., Mercer, N. \& Dawes, L. (1999). From social interaction to individual reasoning: An empirical investigation of a possible sociocultural model of cognitive development. Learning and Instruction, 9, 493-516. https://doi.org/10.1016/ S0959-4752(99)00013-4

Yang, Y. (2016). Lessons learnt from contextualizing a UK teaching thinking program in a conventional Chinese classroom. Thinking Skills and Creativity, 19, 198-209. https://doi.org/10.1016/j. tsc.2015.07.002

Zapiti, A. \& Psaltis, C. (2012). Asymmetries in peer interaction: The effect of social representations of gender and knowledge asymmetry on children's cognitive development. European Journal of Social Psychology, 42, 578-588. https://doi.org/10.1002/ ejsp. 1885

Recibido: 11 de Julio, 2017 Revisado: 11 de octubre, 2017 Aceptado: 23 de octubre, 2017 